\title{
Methods of Improving Productivity in Apparel Industry
}

\author{
R. Subhashini ${ }^{1 *}$, Nirmala Varghese ${ }^{2}$ \\ ${ }^{1,2}$ Department of Apparel and Fashion Design, PSG College of Technology, Coimbatore, India
}

\begin{abstract}
Productivity plays an important role in any garment industry. There are various reasons for which the productivity decreases and there are many known methods to improve the productivity. Choosing a correct and effective method plays a crucial role. A garment manufacturing unit is considered for study and analysis. The study helped in identifying the problems in the industry. The study reported that productivity is low due to time Study analysis not being followed for the sewing operations. Secondly, employees are not motivated in the workplace. In order to improve the productivity, time study and motion study are the effective methods. Work study is known to be a highly effective productivity improvement method. Determining the standard work cycle time is one of the most important step used in work study, which helps in great deal in improving activities. The time study and method study was carried out and was found that the employees are not motivated in the workplace and that has resulted in low productivity and efficiency. Subsequently with the help of management various monetary and non-monetary incentives to employees were suggested in addition to salary on weekly or monthly basis. The introduction of the incentive schemes has motivated the employees to work more efficiently. The time and motion study carried out in the industry has improved the productivity by $33 \%$ and on an average; an operator makes 32 pieces per hour.
\end{abstract}

Keywords: Time study, Motion study, Work study, Flow process chart, Time standards, Productivity, Employee motivation, Incentive schemes.

\section{Introduction}

The study was carried out at a garment industry "Lango Garments". It is established in the year 2007.The industry produces 15,000 garments per day and has well-equipped inhouse factory of 40,000 sq. ft. operating with 150 machinery.

The productivity of the industry was studied and it was observed that the time study analysis is not being followed for the sewing operations. Hence, the study aims to identify the bottleneck operations and suggest appropriate methods to improve the productivity of the industry to overcome the problems.

Productivity is obtained by dividing output by one of the factors of production. Work study may be a method of enhancing the productivity of the firm by eliminating the waste and unnecessary operations. It identifies non-value adding operations by analyzing all the factors affecting the operation. Method study and work measurement are a part of time and motion study. Work measurement is additionally called by the name 'Time study'. Work measurement is completely essential for both the design and control of operations. Highly motivated employees focus their efforts on achieving the precise goals. It's the manager's job, therefore, to motivate the employees to get them to take up and try the simplest job. Highly motivated employees call in sick less frequently, are more productive, and are less likely to convey bad attitudes to customers and associates. The motivation given has to be justified and should be rational. It should be ready to fulfill the aim of productivity improvement otherwise it's going to result into huge loss to the organization and should even cause unethical work practices. Rewards and incentives within the workplace have benefits for both employees and employers. These incentives encourage friendly competition between workers when linked to job performance. The sharing of a company's profits gives incentive to employees to supply a top quality product, perform a top quality service, or improve the standard of a process within the corporate. Non-financial incentives help in satisfying psychological, social and emotional needs of the workers. Few examples for incentives include attendance bonus, group incentives, and monetary awards, allow flexibility and allowances, giving an opportunity to steer, work-life balance, helpful services, healthy food etc.

\section{Literature Review}

\section{A. Productivity}

According to J. R. Hicks, "Production is an activity whether physical or mental, which is directed to the satisfaction of other people's wants through exchange. "Productivity is defined as the ratio between output and input. Output determines the quantity produced and input determines the various resources employed like man, material, machines etc., (Ramesh Babu2012). Productivity refers to the efficiency of production system. It is an indicator of how well the factors of production are utilized. (Glock and Kunz-2005).

$$
\text { Productivity }=\text { Output/Input }
$$

\section{B. Work Study}

"Work study is a generic term for those techniques, method study and work measurement which are used in the examination 
of human work in all its contexts. And which lead systematically to the investigation of all the factors which affect the efficiency and economy of the situation being reviewed, in order to effect improvement." (George Kanawaty, 1955).

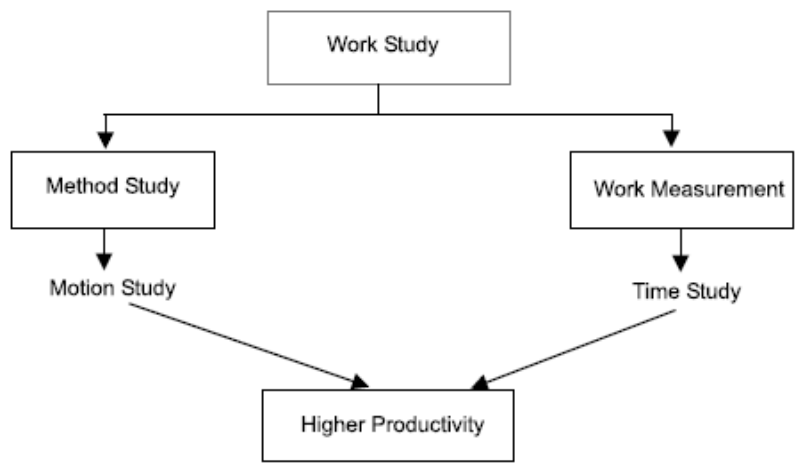

Fig. 1. Framework of work study

\section{1) Method study}

"Method study is the systematic recording and critical examination or existing and proposed ways of doing work as a means or developing and applying easier and more effective methods and reducing cost." (George Kanawaty, 1955).

2) Recording techniques for method study

Following the selection of the work to be analyzed, the next step in the basic procedure is to record all details pertaining to the existing method. It is important to provide a method for tracking all the required information regarding the current method in order to imagine the activities chosen for investigation in their entirety and to improve them by subsequent critical review. Records are extremely useful for comparing the success of the proposed improved process before and after.

3) Types of charts

It can be broadly divided into

i. Macro motion charts

ii. Micro motion charts.

\section{i. Macro motion charts:}

Following four charts are used under this type:

\section{Operation Process Chart}

It's also known as an outline phase diagram. By documenting only, the main operations and inspections involved in the process, an activity process map provides a bird's eye view of the entire process.

2. Flow Process Chart

The flow process map depicts the flow of work for a product or a portion of it through a work center or department, with activities recorded using appropriate symbols. It is an amplification of the operation process map, which depicts operations such as inspection, storage, delay, and transportation.

\section{Two Handed Process Chart}

The most accurate form of flow chart is a two-handed (operator process chart), in which the movements of the workers' hands are reported in relation to one another. The twohanded method map is typically limited to work done at a single location. This also provides a graphical representation of the worker's manual tasks in a coordinated manner.

\section{Multiple Activity Chart}

It's a graph in which the actions of multiple subjects (worker or equipment) are reported on a standard time scale to demonstrate their interdependence.

4) Motion study

Motion research is a form of process study in which the motion of an operator or a piece of work is examined using the prescribed methods.

\section{5) Time study}

Work measurement is another name for time study. It is needed for both operational planning and control. Time research is described by the British Standard Institute as "the application of techniques designed to determine the time required for a skilled worker to perform a specified job at a defined level of efficiency." (Glock and Kunz, 2005).

\section{Employee Motivation}

Motive is described by Webster's Dictionary as "something that causes an individual to act." Employee motivation refers to how dedicated an employee is to his or her job, how involved he or she feels in the company's goals, and how motivated he or she feels in their everyday tasks.

Employees who are highly motivated concentrate their energies on achieving particular objectives. As a result, it is the manager's responsibility to inspire workers and encourage them to do their best work. Employees who are motivated are less likely to call in sick, are more active, and are less likely to express negative attitudes to customers and coworkers. Job motivation may be extrinsic or intrinsic, which means that an employee's motivation stems from within. (Stephen, 2016)

\section{1) Incentives}

Despite the promotion of alternative motivators, money continues to play a significant role in the mix of motivators. Employees who participate in benefit sharing receive a share of the profit in addition to their daily pay.

Employees and employers alike benefit from job bonuses and rewards. When linked to job results, these incentives promote friendly competition among associates. Employees are motivated to create a high-quality product, provide high-quality service, or increase the quality of a company's process by sharing profits.

Non-monetary benefits are those that help workers meet their psychological, social, and emotional needs. Attendance bonuses, community rewards, and cash prizes are only a few examples of incentives. Others include allowing flexibility and allowances, providing a chance to lead, promoting work-life balance, providing supportive resources, and providing nutritious food.

\section{Methodology}

Production in the apparel manufacturing industry plays a major role in deciding the profit/loss of the company. There are various reasons that affect the target output or production. Identifying the reasons troubling productivity and the methods used to overcome the problems, by utilizing the available 
manpower in a more effective way are discussed in methodology.

In order to achieve the objectives of the project, the methodology flow chart has been designed. The methodology followed in the study is explained in the Figure 2 and 3.

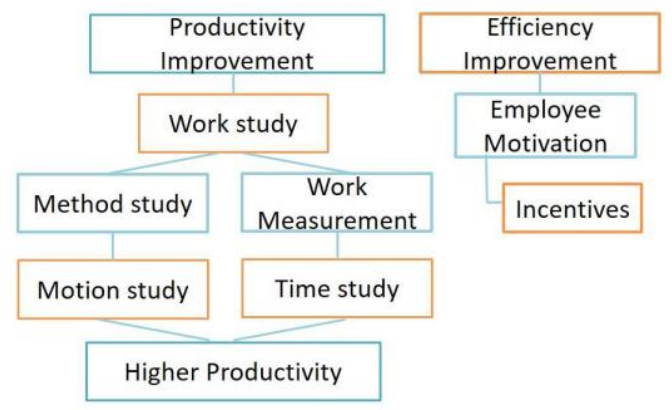

Fig. 2. Methodology outline chart

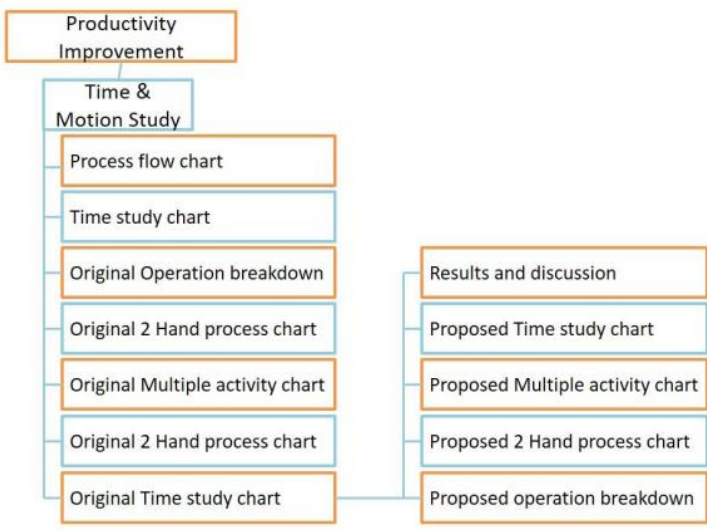

Fig. 3. Methodology flowchart

\section{A. Productivity Improvement}

In order to improve the productivity in the industry following methods are followed.

1) Style Analysis

This polo T-shirt shown in Figure 4 is exclusively designed for men's upon considering the comfort. It is made up of $100 \%$ cotton, Pique fabric with 160 GSM. It has sizes from M, L, and $\mathrm{XL}$. This polo T-shirt has the following parts,
a. Front part
b. Back part
c. Sleeves - 2 No's
d. Collar
e. Placket
f. Side label
g. Contrast cuff

\section{2) Process Flow Chart and Operation Breakdown Table}

A process analysis can be used to improve understanding of how the process operates and to determine potential targets for the process improvement through removing waste. Thus, the chart shows the flow of operations which needs to be done in order to complete the sewing of the polo neck T-shirt. It shows the process flow of both machine and non- machine operations of each part of the polo T-shirt. Process layout of polo T-shirt is shown in Table 1. The stitches and machines required to construct Polo Neck T-shirt is shown in Table 2.

Table 1

Process layout of polo-shirt

\begin{tabular}{|c|l|c|c|c|}
\hline SL. & Operation & M/C & \multicolumn{2}{|c|}{ Man power } \\
\cline { 4 - 5 } No. & & & Helper & Operator \\
\hline 1 & Marking placket position on front panel & Helper & 1 & \\
\hline 2 & Placket rolling & Helper & 1 & \\
\hline 3 & Placket cutting & Helper & 1 & \\
\hline 4 & Placket joint in front panel & SNLS & & 1 \\
\hline 5 & Nose tack of placket in front panel & SNLS & & 1 \\
\hline 6 & Finishing the edges & OL & & 1 \\
\hline 7 & Body match of placket & Helper & 1 & \\
\hline 8 & Shoulder joint & SNLS & & 1 \\
\hline 9 & Shoulder top stitch & SNLS & & 1 \\
\hline 10 & Care label joint & SNLS & & 1 \\
\hline 11 & Collar marking to front panel & Helper & 1 & \\
\hline 12 & Collar joining & SNLS & & 1 \\
\hline 13 & Collar over locking & OL & & 1 \\
\hline 14 & Neck Piping & FL & & 1 \\
\hline 15 & Neck top bound stitch & SNLS & & 1 \\
\hline 16 & Main label joint to neck & SNLS & & 1 \\
\hline 17 & Lower placket finish & SNLS & & 1 \\
\hline 18 & Upper placket finish & SNLS & & 1 \\
\hline 19 & Placket tack (upper \& lower placket finishing) & SNLS & & 1 \\
\hline 20 & Placket trimming & Helper & 1 & \\
\hline 21 & Placket front box making & SNLS & & 1 \\
\hline 22 & Body panel hemming & OL & & 1 \\
\hline 23 & Side band seaming & OL & & 1 \\
\hline 24 & Sleeve matching & Helper & 1 & \\
\hline 25 & Shoulder piping & Helper & 1 & \\
\hline 26 & Sleeve join seam & OL & & 1 \\
\hline 27 & Side band tacking & SNLS & & 1 \\
\hline 28 & Side band top stitch & SNLS & & 1 \\
\hline 29 & Side seam & OL & & 1 \\
\hline 30 & Side Label seam & OL & & 1 \\
\hline 31 & Sleeve tack & SNLS & & 1 \\
\hline & & & 8 & 23 \\
\hline & & & Total Man Power \\
\cline { 3 - 5 } & & & 31 \\
\hline & & & \\
\hline
\end{tabular}

Table 2

Stitches and machines needed to make Polo T-shirt

\begin{tabular}{|c|l|l|c|}
\hline $\begin{array}{c}\text { SL. } \\
\text { No. }\end{array}$ & Operations & Stitch Type & Machines \\
\hline 1 & Placket Rolling & 1N Lock Stitch & \\
\hline 2 & Placket Join & 1N Lock Stitch & SNLS \\
\hline 3 & Nose Tuck & 1N Lock Stitch & SNLS \\
\hline 4 & Shoulder Join seam & Over edge Stitch & SNLS \\
\hline 5 & Collar Join seam & 1N Lock Stitch & OL \\
\hline 6 & Collar Finishing & Over edge Stitch & SNLS \\
\hline 7 & Upper Placket Top stitch & 1N Lock Stitch & OL \\
\hline 8 & Lower Placket Top stitch & 1N Lock Stitch & SNLS \\
\hline 9 & Back Neck Top stitch & 1N Lock Stitch & SNLS \\
\hline 10 & Placket top stitch & 1N Lock Stitch & SNLS \\
\hline 11 & Placket Box stitch & 1N Lock Stitch & SNLS \\
\hline 14 & Sleeve Cuff Joining & Over edge Stitch & SNLS \\
\hline 15 & Cuff Top Stitch & Chain / Flat bed Stitch & OL \\
\hline 16 & Sleeve Joining & Over edge Stitch & FL \\
\hline 17 & Arm Hole Top stitch & Chain / Flat bed Stitch & OL \\
\hline 18 & Side Seam & Over edge Stitch & FL \\
\hline 19 & Body panel Heming & Flat lock & OL \\
\hline 20 & Sleeve tuck seam & 1N Lock Stitch & FL \\
\hline 21 & Button Hole stitch & Button Holing & SNLS \\
\hline 22 & Button seam & Button Attaching & BH \\
\hline 23 & Side label & Label seam & BA \\
\hline & & Total Machine & OL \\
\hline
\end{tabular}




\section{3) SAM allocation}

Based on the motion study and cycle time of an operation, a standard time is allocated for every operation known as SMV (standard minute value). Each operation was measured in seconds and is recorded. Time study sheet is showing the different types of processes, standard time for each operation in minutes, with allowance added 15\%, SMV and SAM for Polo T-shirt. Table 3 shows the operation breakdown with SMV.

\section{Table 3}

Operation breakdown with SMV for Polo T- shirt

\begin{tabular}{|c|c|c|c|}
\hline $\begin{array}{l}\text { SL. } \\
\text { No. }\end{array}$ & Operation & $\begin{array}{c}\text { Standard } \\
\text { Time (sec) }\end{array}$ & $\begin{array}{c}\text { Allowance } \\
15 \%\end{array}$ \\
\hline 1 & Marking placket position & 26 & 3.9 \\
\hline 2 & Placket rolling & 36 & 5.4 \\
\hline 3 & Placket cutting & 28 & 4.2 \\
\hline 4 & Placket joint in front panel & 62 & 9.3 \\
\hline 5 & Nose tack of placket in front panel & 25 & 3.75 \\
\hline 6 & Finishing the edges & 26 & 3.9 \\
\hline 7 & Body match of placket & 20 & 3 \\
\hline 8 & Shoulder joint & 50 & 7.5 \\
\hline 9 & Shoulder top stitch & 44 & 6.6 \\
\hline 10 & Care label joint & 22 & 3.3 \\
\hline 11 & Collar marking to front panel & 30 & 4.5 \\
\hline 12 & Collar joining & 40 & 6 \\
\hline 13 & Collar over locking & 36 & 5.4 \\
\hline 14 & Neck Piping & 38 & 5.7 \\
\hline 15 & Neck top bound stitch & 55 & 8.25 \\
\hline 16 & Main label joint to neck & 32 & 4.8 \\
\hline 17 & Lower placket top seam finish & 44 & 6.6 \\
\hline 18 & Upper placket top seam finish & 54 & 8.1 \\
\hline 19 & Placket tack (upper \& 1ower placket finishing) & 32 & 4.8 \\
\hline 20 & Placket trimming & 21 & 3.15 \\
\hline 21 & Placket front box making & 31 & 4.65 \\
\hline 22 & Body panel hemming & 32 & 4.8 \\
\hline 23 & Side band seaming & 36 & 5.4 \\
\hline 24 & Sleeve matching & 30 & 4.5 \\
\hline 25 & Shoulder piping & 20 & 3 \\
\hline 26 & Sleeve join seam & 45 & 6.75 \\
\hline 27 & Side band tacking & 40 & 6 \\
\hline 28 & Side band top stitch & 35 & 5.25 \\
\hline 29 & Side seam & 40 & 6 \\
\hline 30 & Side label seam & 15 & 2.3 \\
\hline 31 & Sleeve tack & 20 & 3 \\
\hline \multicolumn{2}{|c|}{ Tota1 Estimated SMV } & $17.75(\min )$ & $2.66(\min )$ \\
\hline
\end{tabular}

No. of Operator: 31

Standard Minute Value (SMV): 17.75 (min)

$\mathrm{SAM}=(\mathrm{SMV}+15 \%$ allowance $)$

$=17.45+2.66=20.41(\mathrm{~min})$

Target per hour: $(31 \times 60) / 20.41=91.13 \mathrm{pcs} / \mathrm{hr}$.

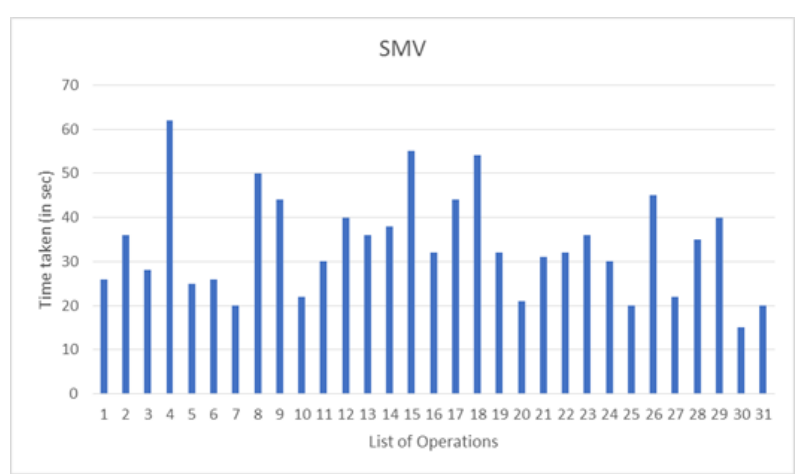

Fig. 5. Estimated standard minute values

The Figure 5 shows that there are thirty-one (31) operation breakdowns where most of the values for SMV are below 50 . The target per hour of a qualified operator will be 91 pieces. On the other hand, operation SMV above 60, are observed as placket finishing, and side seam operation which are 220 and 130 seconds respectively when the whole operation is combined.

\section{4) The Original Method}

An operation of placket making with side seam of a Polo Tshirt has been taken and it is required to study the existing original method using work study and thereby propose a new method which is more efficient and less time consuming. The existing method is the traditional method in practice for making polo T-shirt front placket and side seam which is followed.

\section{a) Operational breakdown for placket}

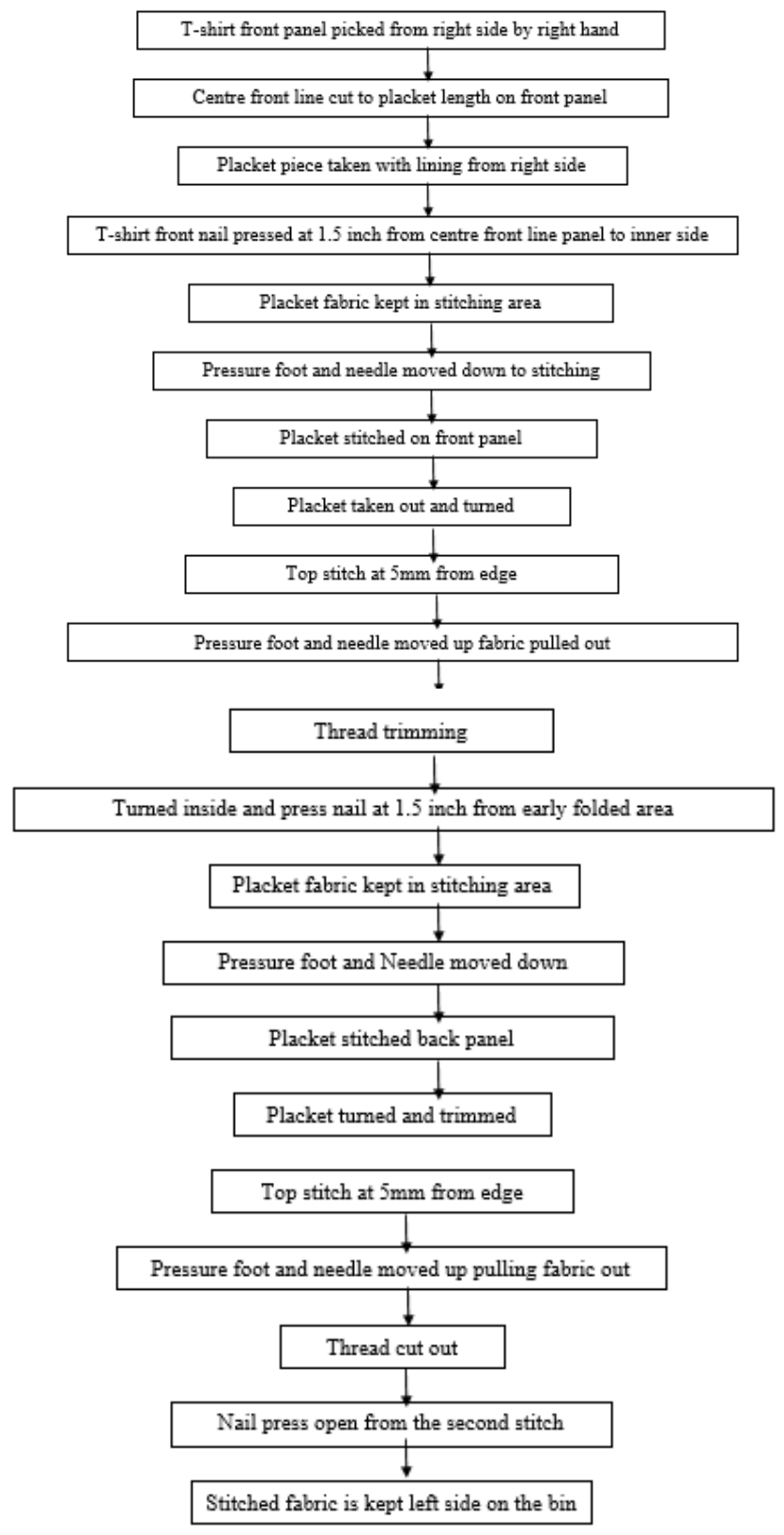


b) Operational breakdown for side seam

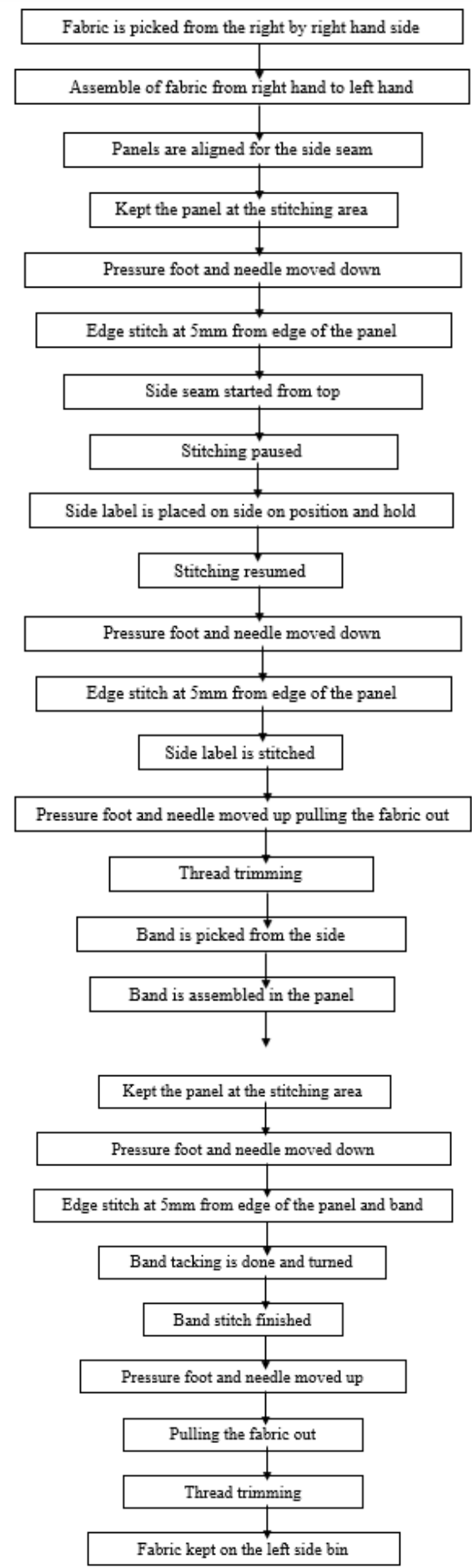

c) Two handed process chart (for placket)

A Two handed process chart for placket is shown in Table 4.

\begin{tabular}{|c|c|c|c|c|c|c|c|c|c|}
\hline Left Hand Side & 0 & $\Rightarrow$ & $D$ & $\nabla$ & O & $\Rightarrow$ & $D$ & $\nabla$ & 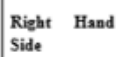 \\
\hline Idle & 0 & द) & 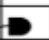 & $\nabla$ & 9 & $\Rightarrow$ & $D$ & $\nabla$ & Froat picked \\
\hline Hold front & 6 & $\Rightarrow$ & $D$ & $\nabla$ & 9 & $\Rightarrow$ & $D$ & $\nabla$ & \begin{tabular}{|l|l}
$\begin{array}{l}\text { Marking } \\
\text { placket }\end{array}$ for \\
pol
\end{tabular} \\
\hline Hold frent & 0 & $\Rightarrow$ & D & $\nabla$ & 9 & $\Rightarrow$ & $D$ & $\nabla$ & $\begin{array}{l}\text { Frout panel cut } \\
\text { for placket }\end{array}$ \\
\hline Idle & 0 & $\Rightarrow$ & D & $\nabla$ & 9 & $\Rightarrow$ & $D$ & $\nabla$ & $\begin{array}{l}\text { Placket piece } \\
\text { taken }\end{array}$ \\
\hline Idle & 0 & $\Rightarrow$ & D & $\nabla$ & 9 & $\Rightarrow$ & $D$ & $\nabla$ & $\begin{array}{l}\text { Plackent and } \\
\text { panel aligned }\end{array}$ \\
\hline
\end{tabular}

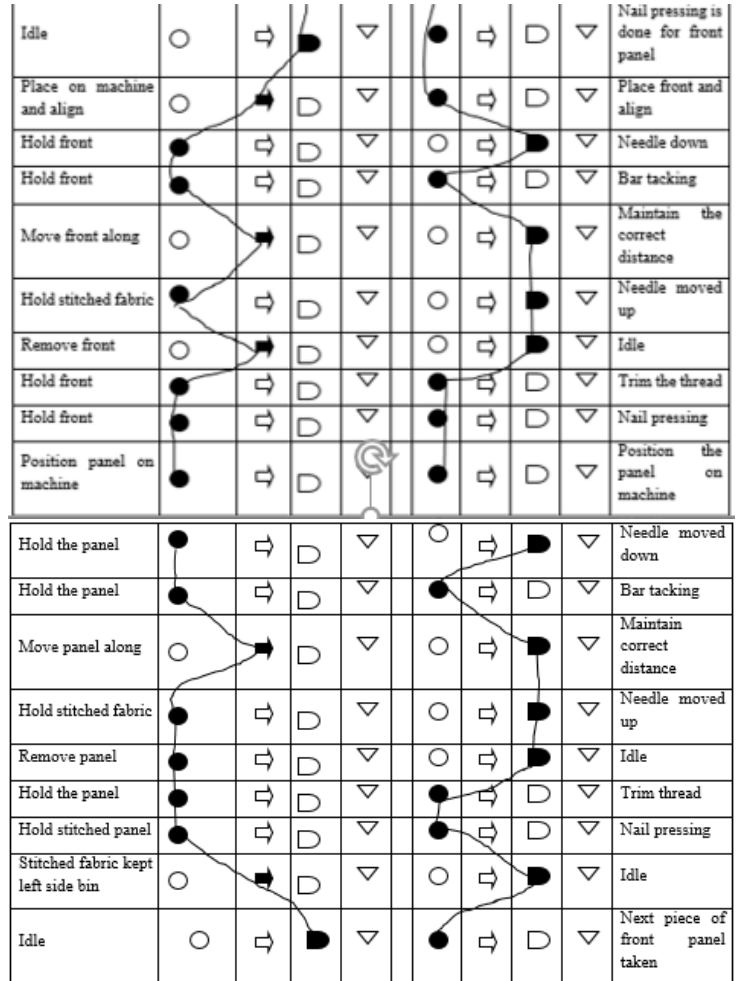

d) Two handed process chart (for side seam)

A two handed process chart for side seam is shown in Table 5 .

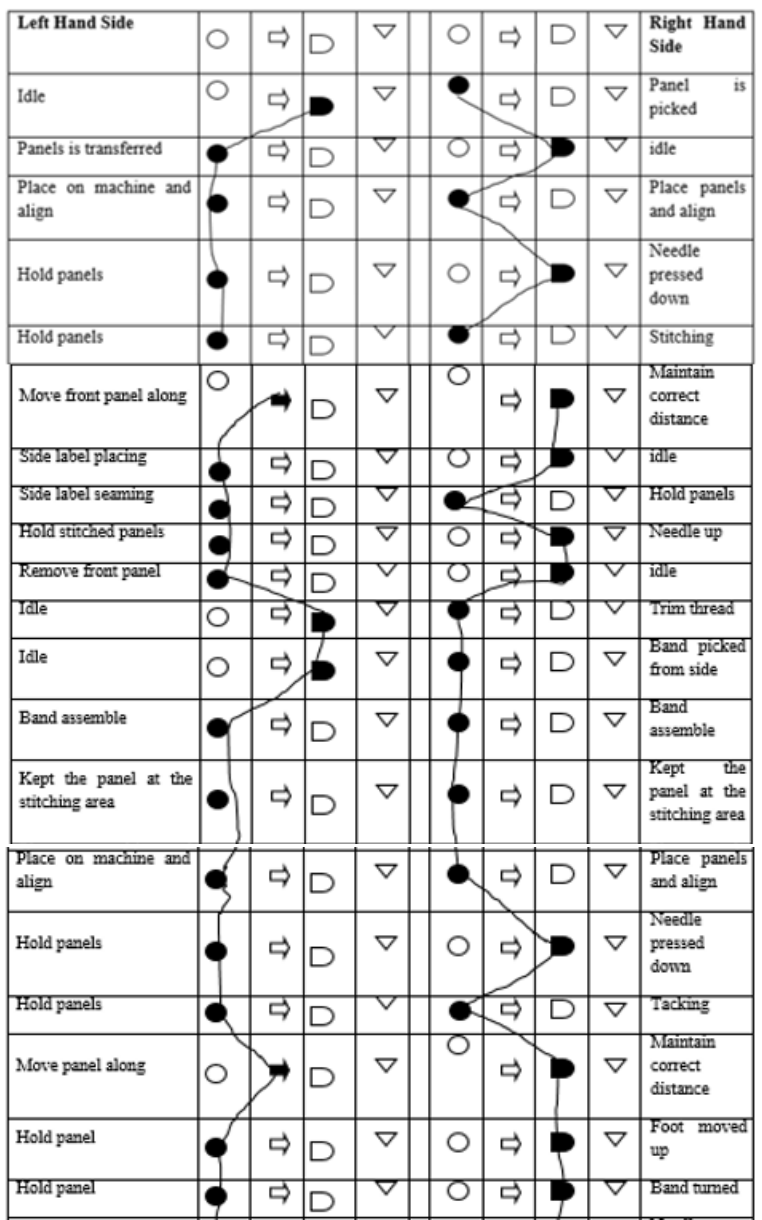




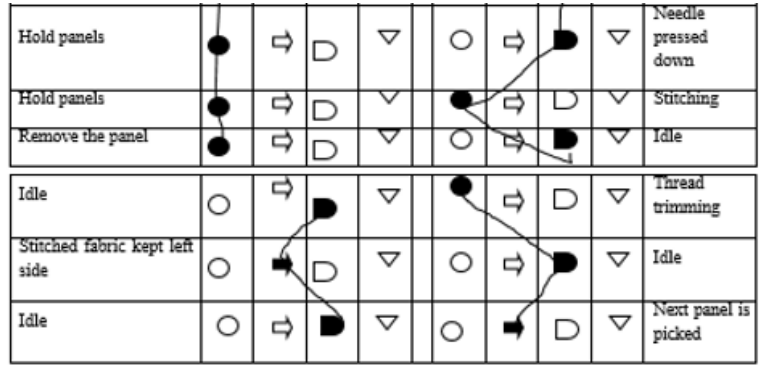

e) Multiple Activity Chart for Placket

A multiple activity chart for placket is shown in Table 6.

Table 6

Multiple activity chart for placket

\begin{tabular}{|l|l|}
\hline MAN & MACHINE \\
\hline Front picked & Idle \\
\hline Marking for placket & Idle \\
\hline Front panel cut for placket & Idle \\
\hline Placket piece taken & Idle \\
\hline Placket and panel aligned & Idle \\
\hline Nail pressing is done for front panel & Idle \\
\hline Panel placed it in stitching area & idle \\
\hline pressure foot \& needle down & idle \\
\hline Idle & Bar-tack, stitch on 5 mm from edge \\
\hline Idle & Stitching complete \\
\hline Pressure foot and needle moved up & idle \\
\hline Remove fabric from machine & Idle \\
\hline Trim the thread & Idle \\
\hline Nail press at 1.5 inch & idle \\
\hline Place fabric in the stitching area & Idle \\
\hline Pressure foot and needle put down & idle \\
\hline Idle & Bar tack, stitch on 5 mm from edge \\
\hline Idle & Stitch complete \\
\hline Pressure foot and needle moved up & idle \\
\hline Remove and discard the panel & Idle \\
\hline Nail press open at second seam & idle \\
\hline Place the stitched fabric at other side & Idle \\
\hline
\end{tabular}

\section{f) Multiple Activity Chart for Side Seam}

A multiple activity chart for side seam is shown in Table 7.

Table 7

Multiple activity chart for side seam

\begin{tabular}{|l|l|}
\hline MAN & MACHINE \\
\hline Panel picked & Idle \\
\hline Panel placed at side seam & Idle \\
\hline Panel placed it in stitching area & idlle \\
\hline Pressure foot and needle moved down & idlle \\
\hline Idlle & Bar-tack, stitch on $5 \mathrm{~mm}$ from edge \\
\hline Idlle & Stitching complete \\
\hline Pressure foot and needle moved up & idle \\
\hline Remove fabric from the machine & Idle \\
\hline Trimming & Idle \\
\hline Band picked from side & Idle \\
\hline Band assemble & Idlle \\
\hline Kept the panel at the stitching area & Idlle \\
\hline
\end{tabular}

\begin{tabular}{|l|l|}
\hline Place on machine and align & Idle \\
\hline Hold the fabric & Tacking \\
\hline Band turned & Idle \\
\hline Stitching & Idle \\
\hline Remove fabric from the machine & Idle \\
\hline Thread trimming & Idle \\
\hline Place the stitched fabric at other bin & Idlle \\
\hline Next panel is picked & Idle \\
\hline
\end{tabular}

g) Time Study Chart for Placket

A time study chart for placket is shown in Table 8.

Table 8

Time study chart for placket

\begin{tabular}{|c|c|c|c|c|c|c|c|}
\hline & & TIMI & STUD & CHA & & & \\
\hline $\begin{array}{l}\mathrm{OPE} \\
\mathrm{MAC}\end{array}$ & $\begin{array}{l}\text { ATION NAME: } \\
\text { IINE USED: } \\
\text { SNLS }\end{array}$ & Making & & & & & \\
\hline No & Operation & Obse & $\operatorname{ved~} \mathrm{Ti}$ & & Total & Avg & Total \\
\hline & Operatuon & 1 & 2 & 3 & SMV & SMV & SMV \\
\hline 1 & \begin{tabular}{|l|} 
T-shirt front panel \\
picked from right side \\
by right hand
\end{tabular} & 1.2 & 1 & 1.5 & 3.7 & 1.2 & \begin{tabular}{|l|l|}
1.23 & \\
\end{tabular} \\
\hline 2 & $\begin{array}{l}\text { Centre front line cut to } \\
\text { placket length on front } \\
\text { panel }\end{array}$ & 3 & 3.5 & 3.4 & 9.9 & 3.3 & 3.30 \\
\hline 3 & $\begin{array}{l}\text { Placket piece taken with } \\
\text { lining from right side }\end{array}$ & 1.2 & 1 & 1.5 & 3.7 & 1.2 & 1.23 \\
\hline 4 & $\begin{array}{l}\text { T-shirt front nail pressed } \\
\text { at } 1.5 \text { inch from centre } \\
\text { front line panel to inner } \\
\text { side }\end{array}$ & 8 & 8.5 & 8 & 24.5 & 8.17 & 8.17 \\
\hline 5 & $\begin{array}{l}\text { Placket fabric kept in } \\
\text { stitching area }\end{array}$ & 2 & 2.3 & 2.2 & 6.5 & 2.17 & 2.17 \\
\hline 6 & $\begin{array}{l}\text { Pressure foot and needle } \\
\text { moved down to stitching }\end{array}$ & 1 & 1.2 & 1 & 3.2 & 1.07 & \begin{tabular}{ll|l|l|}
1.07 &
\end{tabular} \\
\hline 7 & \begin{tabular}{|l|}
$\begin{array}{l}\text { Placket stitched on front } \\
\text { panel }\end{array}$ \\
\end{tabular} & 15 & 14.8 & 15.5 & 45.3 & 15.1 & 15.10 \\
\hline 8 & \begin{tabular}{|l|}
$\begin{array}{l}\text { Placket taken out and } \\
\text { turned }\end{array}$ \\
\end{tabular} & 5 & 5.5 & 5.5 & 16 & 5.33 & 5.33 \\
\hline 9 & \begin{tabular}{|l|}
$\begin{array}{l}\text { Top stitch at } 5 \mathrm{~mm} \text { from } \\
\text { edge }\end{array}$
\end{tabular} & 21 & 20 & 22 & 63 & 21 & 21.00 \\
\hline 10 & $\begin{array}{l}\begin{array}{l}\text { Pressure foot and needle } \\
\text { moved up fabric pulled } \\
\text { out }\end{array} \\
\end{array}$ & 3.5 & 3 & 3.2 & 9.7 & 3.23 & 3.23 \\
\hline 11 & Thread trimming & 2.3 & 2 & 2.2 & 6.5 & 2.17 & 2.17 \\
\hline 12 & $\begin{array}{l}\text { Turned inside and press } \\
\text { nail at } 1.5 \text { inch from } \\
\text { early folded area }\end{array}$ & 12 & 15 & \begin{tabular}{|l|l|}
14.7 & \\
\end{tabular} & 41.7 & 13.9 & 13.90 \\
\hline 13 & $\begin{array}{l}\text { Placket fabric kept in } \\
\text { stitching area }\end{array}$ & 3 & 3.7 & 3.3 & 10 & 3.33 & 3.33 \\
\hline 14 & $\begin{array}{l}\text { Pressure foot and } \\
\text { Needle moved down }\end{array}$ & 1 & 1.2 & 1.2 & 3.4 & 1.13 & 1.13 \\
\hline 15 & $\begin{array}{l}\text { Placket stitched back } \\
\text { panel }\end{array}$ & 15.5 & 14 & 15 & 44.5 & 14.83 & 14.83 \\
\hline 16 & \begin{tabular}{|l}
$\begin{array}{l}\text { Placket } \\
\text { trimmed }\end{array}$ \\
\end{tabular} & 5 & 4.8 & 5.5 & 15.3 & 5.1 & 5.10 \\
\hline 17 & $\begin{array}{l}\text { Top stitch at } 5 \mathrm{~mm} \text { from } \\
\text { edge }\end{array}$ & 22 & 21 & 21 & 64 & 21.33 & 21.33 \\
\hline 18 & $\begin{array}{l}\begin{array}{l}\text { Pressure foot and needle } \\
\text { moved up pulling fabric } \\
\text { out }\end{array} \\
\end{array}$ & 3 & 3.5 & 3.5 & 10 & 3.33 & 3.33 \\
\hline 19 & Thread cut out & 2.2 & 2.2 & 2.5 & 6.9 & 2.3 & 2.30 \\
\hline 20 & $\begin{array}{l}\text { Nail press open from } \\
\text { stitch }\end{array}$ & 8.7 & 7.7 & 7.2 & 23.6 & 7.87 & 7.87 \\
\hline 21 & $\begin{array}{l}\text { Stitched fabric is kept } \\
\text { left side on the bin }\end{array}$ & 1.5 & 1.2 & 1 & 3.7 & 1.23 & 1.23 \\
\hline & & & & & & Total & 138 \\
\hline
\end{tabular}

Calculations:

Standard Minute Value (SMV): $138 \mathrm{sec}$

Calculation of SAM: 
Allowance: $15 \%$

SAM (Standard Allowed Minutes) $=$ Basic Time + Total Allowances

$\mathrm{SAM}=(\mathrm{SMV}+15 \%$ allowance $)$

$=138+15 \%$

$=138+21$

$=159 \mathrm{Sec}$

Therefore, $\mathrm{SAM}=159 \mathrm{sec}=2.6 \mathrm{~min}$

Maximum output $=($ Working time $/$ Maximum process time $)$

$=(60 / 2.6)$

$=23$ Pcs

Productivity per Operator $=$ Total output $/$ Total manpower

$=23$ Pcs $/ 21$ Operator

$=1.09 \mathrm{Pcs} /$ Operator is the output.

h) Time Study Chart for Side Seam

A time study chart for side seam is shown in Table 9.

Table 9

Time study chart for side seam

\begin{tabular}{|c|c|c|c|c|c|c|c|}
\hline \multicolumn{8}{|c|}{ TIME STUDY CHART } \\
\hline \multicolumn{8}{|c|}{$\begin{array}{ll}\text { OPERATIONNAME: } & \text { SIDE SEAM } \\
\text { MACHINE USED: } & \text { SNLS }\end{array}$} \\
\hline No & Operation & \multicolumn{3}{|c|}{ Observed Time } & $\begin{array}{l}\text { Total } \\
\text { SMV }\end{array}$ & $\begin{array}{l}\text { Arg } \\
\text { SMN } \\
\end{array}$ & \begin{tabular}{|l|} 
Total \\
SMV
\end{tabular} \\
\hline 1 & $\begin{array}{l}\text { Fabric is picked from } \\
\text { the right side by right } \\
\text { hand }\end{array}$ & 3 & 3.3 & 3.2 & 9.5 & 3.17 & 3.17 \\
\hline 2 & $\begin{array}{l}\text { Assemble of fabric from } \\
\text { right hand to left hand }\end{array}$ & 2.1 & 2.2 & 2.1 & 6.4 & 2.13 & 2.07 \\
\hline 3 & $\begin{array}{l}\begin{array}{l}\text { Panels are aligned for } \\
\text { the side seam }\end{array} \\
\text { te }\end{array}$ & 2 & 2.5 & 2.4 & 6.9 & 2.3 & 2.30 \\
\hline 4 & \begin{tabular}{|l|}
$\begin{array}{l}\text { Kept the panel at the } \\
\text { stitching area }\end{array}$ \\
\end{tabular} & 4.6 & 5.7 & 6 & 16.3 & 5.43 & 5.43 \\
\hline 5 & \begin{tabular}{|l|}
$\begin{array}{l}\text { Pressure foot and needle } \\
\text { moved down }\end{array}$ \\
\end{tabular} & 2.14 & 2.07 & 3 & 7.21 & 2.4 & 2.40 \\
\hline 6 & \begin{tabular}{|l|}
$\begin{array}{l}\text { Edge stitch at } 5 \mathrm{~mm} \text { from } \\
\text { edge of the panel }\end{array}$ \\
\end{tabular} & 6.2 & 7.3 & 6.8 & 20.3 & 6.77 & 6.60 \\
\hline 7 & $\begin{array}{l}\text { Side label is placed on } \\
\text { side on position and } \\
\text { hold }\end{array}$ & 2 & 2.5 & 3 & 7.5 & 2.5 & 2.50 \\
\hline 8 & \begin{tabular}{|l|}
$\begin{array}{l}\text { Pressure foot and needle } \\
\text { moted down }\end{array}$ \\
\end{tabular} & 2.14 & 2.07 & 3 & 7.21 & 2.4 & 2.40 \\
\hline 9 & $\begin{array}{l}\text { Edge stitich at } 5 \mathrm{~mm} \text { from } \\
\text { edge of the panel }\end{array}$ & 30 & 32 & 35 & 97 & 32.3 & 32.33 \\
\hline 10 & Side label is stitched & 6 & 7.5 & 8.8 & 22.3 & 7.43 & 7.43 \\
\hline 11 & $\begin{array}{l}\text { Preasure foot and needlle } \\
\text { moved up pulling the } \\
\text { fabric out }\end{array}$ & 6 & 7.2 & 7.5 & 20.7 & 6.9 & 6.67 \\
\hline 12 & Thread trimming & 5 & 5.1 & 5.2 & 15.3 & 5.1 & 5.10 \\
\hline 13 & $\begin{array}{l}\begin{array}{l}\text { Band is picked from the } \\
\text { side }\end{array} \\
\end{array}$ & 3 & 3.3 & 3.2 & 9.5 & 3.17 & 3.17 \\
\hline 14 & \begin{tabular}{|l|} 
Band is assembled in the \\
panel
\end{tabular} & 2.1 & 2.2 & 2.1 & 6.4 & 2.13 & 2.07 \\
\hline 15 & \begin{tabular}{|l|}
$\begin{array}{l}\text { Kept the panel at the } \\
\text { stitching area }\end{array}$ \\
\end{tabular} & 2 & 2.5 & 2.4 & 6.9 & 2.3 & 2.30 \\
\hline 16 & \begin{tabular}{|l|}
$\begin{array}{l}\text { Preassure foot and needle } \\
\text { moved down }\end{array}$ \\
\end{tabular} & 2.14 & 2.07 & 3 & 7.21 & 2.4 & 2.40 \\
\hline 17 & $\begin{array}{l}\text { Edge stitch at 5mm from } \\
\text { edge of the panel and } \\
\text { band }\end{array}$ & 6 & 7.5 & 8.8 & 22.3 & 7.43 & 7.43 \\
\hline 18 & $\begin{array}{l}\text { Eand tacking is done } \\
\text { and turned }\end{array}$ & 6.5 & 7.2 & 8.3 & 22 & 7.33 & 7.33 \\
\hline 19 & Band stitch finished & 6 & 7.5 & 8.8 & 22.3 & 7.43 & 7.43 \\
\hline 20 & \begin{tabular}{|l|}
$\begin{array}{l}\text { Preassure foot and needle } \\
\text { moved up }\end{array}$ \\
\end{tabular} & 2.14 & 2.07 & 3 & 7.21 & 2.4 & 2.40 \\
\hline 21 & Pulling the fabric out & 4.56 & 5.5 & 4.5 & 14.6 & 4.85 & 4.69 \\
\hline 22 & Thread trimming & 3 & 3.2 & 3.5 & 9.7 & 3.23 & 3.23 \\
\hline 23 & $\begin{array}{l}\text { Fabric kept on the left } \\
\text { side bin }\end{array}$ & 3.3 & 3.5 & 3.7 & 10.5 & 3.5 & 3.50 \\
\hline & & & & & & \begin{tabular}{|l|} 
Total \\
\end{tabular} & 124.36 \\
\hline
\end{tabular}

\section{Calculations:}

Standard Minute Value (SMV): $124.36 \mathrm{sec}$

Calculation of SAM:

Allowance: $15 \%$

SAM (Standard Allowed Minutes) $=$ Basic Time + Total Allowances

$$
\begin{aligned}
& \text { SAM }=(\mathrm{SMV}+15 \% \text { allowance }) \\
& =124.36+15 \% \\
& =124.36+18.65 \\
& =143.01 \mathrm{Sec}
\end{aligned}
$$

Therefore, $\mathrm{SAM}=143.01 \mathrm{sec}=2.4 \mathrm{~min}$

Maximum output $=($ Working time $/$ Maximum process time $)$

$=(60 / 2.4)$

$=25$ Pcs

Productivity per Operator $=$ Total output/Total manpower

$=25 \mathrm{Pcs} / 23$ Operator

$=1.08 \mathrm{Pcs} /$ Operator is the output.

\section{5) The Proposed Methods}

The operation of placket making with side seam of a polo Tshirt was considered and thereby studied the existing original method using work study and hereby proposed a new method which is more efficient and less time consuming.

\section{a) Operational breakdown for placket}

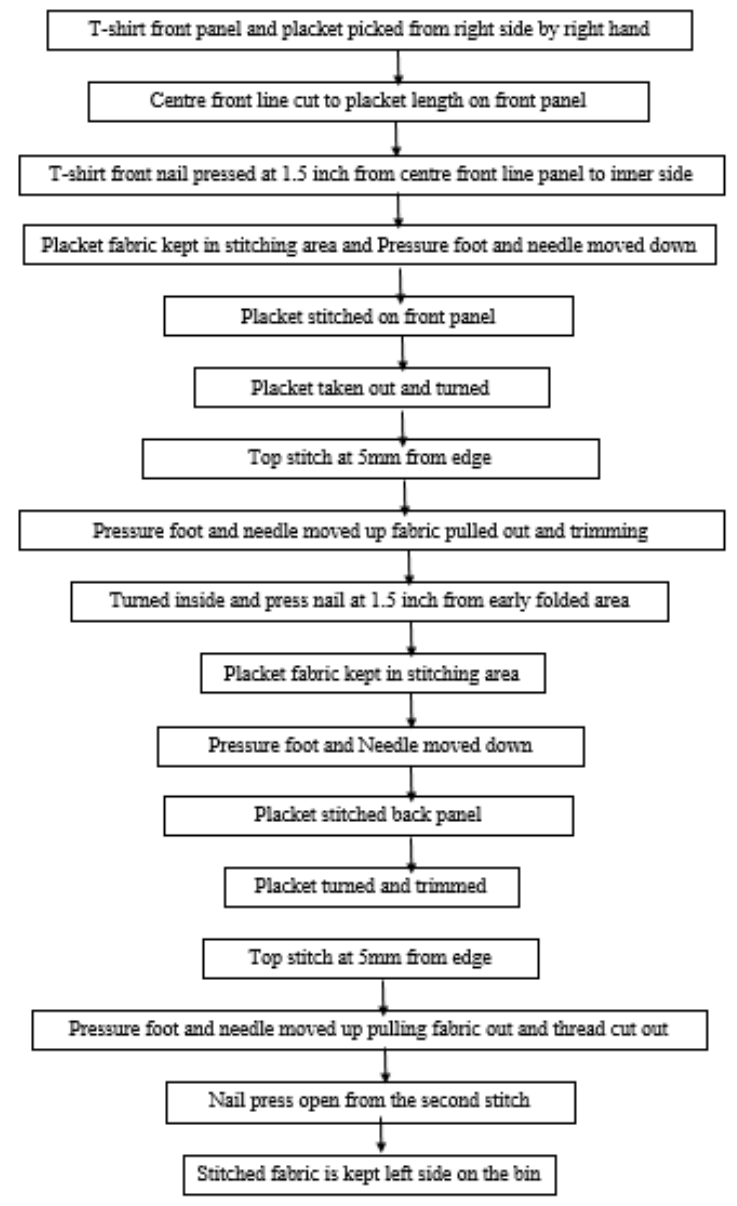




\section{b) Operation breakdown of side seam}

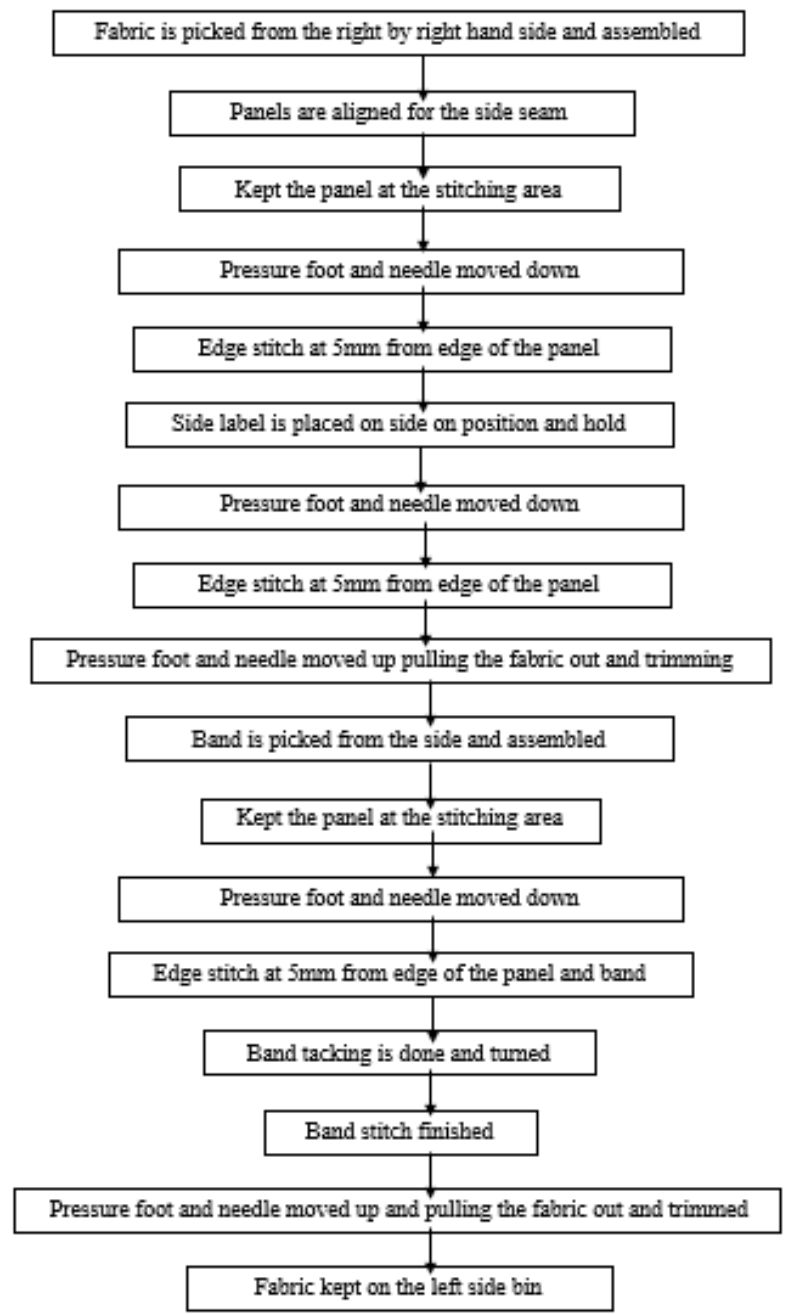

c) Two handed process chart (for placket)

A Two handed process chart for placket is shown in Table 10.

Table 10

Two handed process chart (for placket)

\begin{tabular}{|c|c|c|c|c|c|c|c|c|c|}
\hline Left Hand Side & 0 & $\Rightarrow$ & $D$ & $\nabla$ & 0 & $\Rightarrow$ & $D$ & $\nabla$ & $\begin{array}{l}\text { Right Hand } \\
\text { Side }\end{array}$ \\
\hline Placket picked & & $\Rightarrow$ & $D$ & $\nabla$ & 9 & $\Rightarrow$ & $D$ & $\nabla$ & $\begin{array}{l}\text { Front } \\
\text { picked }\end{array}$ \\
\hline Hold front & & $\Rightarrow$ & $D$ & $\nabla$ & 0 & $\Rightarrow$ & $D$ & $\nabla$ & $\begin{array}{l}\text { Front panel } \\
\text { marking and } \\
\text { cut for placket }\end{array}$ \\
\hline $\begin{array}{l}\text { Placket and panel } \\
\text { aligned }\end{array}$ & & $\Rightarrow$ & $D$ & $\nabla$ & 9 & $\Rightarrow$ & D & $\nabla$ & $\begin{array}{l}\text { Placket and } \\
\text { panel aligned }\end{array}$ \\
\hline Hold front & & $\Rightarrow$ & $D$ & $\nabla$ & & $\Rightarrow$ & $D$ & $\nabla$ & $\begin{array}{l}\text { Nail pressing is } \\
\text { done for front } \\
\text { panel }\end{array}$ \\
\hline $\begin{array}{l}\text { Place on machine } \\
\text { and align }\end{array}$ & 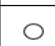 & & $D$ & $\nabla$ & a. & $\Rightarrow$ & $D$ & $\nabla$ & $\begin{array}{l}\text { Place front and } \\
\text { align }\end{array}$ \\
\hline Hold front & & $\Rightarrow$ & $D$ & $\nabla$ & O & $\Rightarrow$ & D & $\nabla$ & Needle down \\
\hline Hold front & & $\Rightarrow$ & $D$ & $\nabla$ & Q & $\Rightarrow$ & $D$ & $\nabla$ & Bar tacking \\
\hline Move front along & & & $D$ & $\nabla$ & O & $\Rightarrow$ & & $\nabla$ & $\begin{array}{l}\text { Maintain the } \\
\text { correct } \\
\text { distance }\end{array}$ \\
\hline Hold stitched fabric & 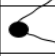 & $\Rightarrow$ & $D$ & $\nabla$ & 0 & $\Rightarrow$ & 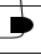 & $\nabla$ & $\begin{array}{l}\text { Needle moved } \\
\text { up }\end{array}$ \\
\hline Remove front & 0 & $\Rightarrow$ & $D$ & $\nabla$ & 9 & $\Rightarrow$ & $D$ & $\nabla$ & Trim the thread \\
\hline Hold folded front & 8 & $\Rightarrow$ & $D$ & $\nabla$ & 6 & $\Rightarrow$ & $D$ & $\nabla$ & Nail pressing \\
\hline Position the panel & 9 & $\Rightarrow$ & $D$ & $\nabla$ & O & $\Rightarrow$ & D & $\nabla$ & $\begin{array}{l}\text { Needle moved } \\
\text { down }\end{array}$ \\
\hline Hold the panel & 2 & $\Rightarrow$ & $D$ & $\nabla$ & $\gamma$ & $\Rightarrow$ & $D$ & $\nabla$ & Bar tacking \\
\hline Move panel along & 0 & $\Rightarrow$ & $D$ & $\nabla$ & 0 & $\Rightarrow$ & D & $\nabla$ & $\begin{array}{l}\text { Maintain } \\
\text { correct } \\
\text { distance }\end{array}$ \\
\hline
\end{tabular}

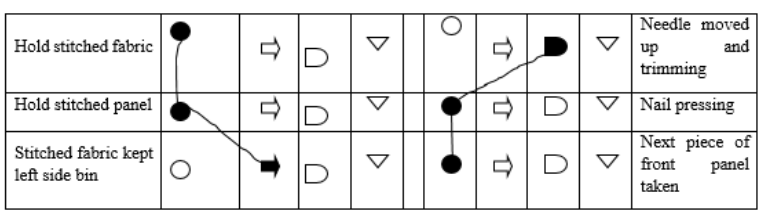

d) Two handed process chart (for side seam)

A Two handed process chart for side seam is shown in Table 11.

Table 11

Two handed process chart (for side seam)

\begin{tabular}{|c|c|c|c|c|c|c|c|c|c|}
\hline \begin{tabular}{|l|} 
Left Hand Side \\
\end{tabular} & 0 & $\Rightarrow$ & $D$ & $\nabla$ & 0 & $\Rightarrow$ & $D$ & $\nabla$ & $\begin{array}{l}\text { Right Hand } \\
\text { Side }\end{array}$ \\
\hline Panels is transferred & O & $\Rightarrow$ & D & $\nabla$ & & $\Rightarrow$ & $D$ & $\nabla$ & $\begin{array}{l}\begin{array}{l}\text { Panel } \\
\text { picked }\end{array} \\
\text { is }\end{array}$ \\
\hline $\begin{array}{l}\text { Place on machine and } \\
\text { align }\end{array}$ & & $\Rightarrow$ & $D$ & $\nabla$ & 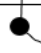 & $\Rightarrow$ & D & $\nabla$ & $\begin{array}{l}\text { Place panels } \\
\text { and align }\end{array}$ \\
\hline Hold panels & & $\Rightarrow$ & $D$ & $\nabla$ & 0 & Б & & $\nabla$ & $\begin{array}{l}\text { Needle } \\
\text { pressed } \\
\text { down }\end{array}$ \\
\hline \begin{tabular}{|l} 
Hold panels \\
\end{tabular} & 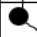 & $\Rightarrow$ & $D$ & $\nabla$ & & $\Rightarrow$ & $D$ & $\nabla$ & \begin{tabular}{|l|} 
Stitching \\
\end{tabular} \\
\hline Move front panel along & 0 & 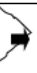 & $D$ & $\nabla$ & 0 & $\Rightarrow$ & & $\nabla$ & $\begin{array}{l}\text { Maintain } \\
\text { correct } \\
\text { distance }\end{array}$ \\
\hline Side label seaming & & $\Rightarrow$ & $D$ & $\nabla$ & & $\Rightarrow$ & D & $\nabla$ & $\begin{array}{l}\text { Side label } \\
\text { placing }\end{array}$ \\
\hline Hold stitched panels & & 다 & $D$ & $\nabla$ & 0 & दे & & $\nabla$ & Needle up \\
\hline Remove front panel & 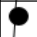 & 다 & $D$ & $\nabla$ & & ᄃ & $D$ & $\nabla$ & Trim thread \\
\hline Band assemble & & 다 & $D$ & $\nabla$ & 0 & $\Rightarrow$ & $D$ & $\nabla$ & $\begin{array}{l}\text { Band picked } \\
\text { from side }\end{array}$ \\
\hline $\begin{array}{l}\text { Kept the panel at the } \\
\text { stitching area and align }\end{array}$ & 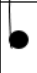 & $\Rightarrow$ & $D$ & $\nabla$ & $b$ & $\Rightarrow$ & D & $\nabla$ & $\begin{array}{l}\text { Kept the } \\
\text { panel at the } \\
\text { stitching area }\end{array}$ \\
\hline Hold panels & & $\Rightarrow$ & $D$ & $\nabla$ & 0 & 다 & & $\nabla$ & \begin{tabular}{|l} 
Needle \\
pressed \\
down
\end{tabular} \\
\hline Move panel along & & & $D$ & $\nabla$ & 0 & a & & $\nabla$ & \begin{tabular}{|l} 
Maintain \\
correct \\
distance
\end{tabular} \\
\hline Hold panel & & 디 & $D$ & $\nabla$ & $\mathrm{O}$ & द & & $\nabla$ & Band turned \\
\hline Hold panels & & 다 & $D$ & $\nabla$ & $\mathrm{O}$ & $\Rightarrow$ & & $\nabla$ & $\begin{array}{l}\text { Needle } \\
\text { pressed } \\
\text { down and } \\
\text { stitching }\end{array}$ \\
\hline Remove the panel & & $\Rightarrow$ & $D$ & $\nabla$ & & $\Rightarrow$ & $D$ & $\nabla$ & \begin{tabular}{|l|} 
Thread \\
trimming
\end{tabular} \\
\hline $\begin{array}{l}\text { Stitched fabric kept left } \\
\text { side }\end{array}$ & $\mathrm{O}$ & $\Rightarrow$ & $D$ & $\nabla$ & 0 & $\Rightarrow$ & $D$ & $\nabla$ & $\begin{array}{l}\text { Next panel is } \\
\text { picked }\end{array}$ \\
\hline
\end{tabular}

e) Multiple Activity Chart for Placket

A multiple activity chart for placket is shown in Table 12.

Table 12

Multiple activity chart for placket

\begin{tabular}{|l|l|}
\hline MAN & MACHINE \\
\hline Front panel and placket picked & Idle \\
\hline $\begin{array}{l}\text { Marking for placket and Front panel cut for } \\
\text { placket }\end{array}$ & Idle \\
\hline Nail pressing is done for front panel & Idle \\
\hline Panel placed it in stitching area & pressure foot \& needle down \\
\hline Idle & Bar-tack, stitch on 5 mm from edge \\
\hline Idle & Stitching complete \\
\hline Remove fabric from machine & Pressure foot and needle moved up \\
\hline Trim the thread & Idle \\
\hline Nail press at 1.5 inch & idle \\
\hline Place fabric in the stitching area & Pressure foot and needle put down \\
\hline Idle & Bar tack, stitch on 5 mm from edge \\
\hline Idle & Stitch complete \\
\hline
\end{tabular}




\begin{tabular}{|l|l|}
\hline Pressure foot and needle moved up & Remove the panel \\
\hline Nail press open at second seam & idle \\
\hline Place the stitched fabric at other side & Idle \\
\hline
\end{tabular}

\section{f) Multiple Activity Chart for Side seam}

A multiple activity chart for side seam is shown in Table 13.

Table 13

Multiple activity chart for side seam

\begin{tabular}{|l|l|}
\hline MAN & MACHINE \\
\hline Panel picked and placed at side seam & Idle \\
\hline Panel placed it in stitching area & Pressure foot and needle moved down \\
\hline Idle & Bar-tack, stitch on 5 mm from edge \\
\hline Idle & Stitching complete \\
\hline Remove fabric from the machine & Pressure foot and needle moved up \\
\hline Trimming & Idle \\
\hline Band picked from side and assemble & Idle \\
\hline Kept the panel at the stitching area and align & Idle \\
\hline Hold the fabric & Tacking \\
\hline Band turned & Stitching \\
\hline Remove fabric from the machine & Idle \\
\hline Thread trimming & Idle \\
\hline Place the stitched fabric at other bin & Idle \\
\hline Next panel is picked & Idle \\
\hline
\end{tabular}

g) Time Study Chart for Placket

A time study chart for placket is shown in Table 14 .

Table 14

Time study chart for placket

\begin{tabular}{|c|c|c|c|c|c|c|c|}
\hline & & TIME & STUDY & CHAR & & & \\
\hline $\begin{array}{l}\mathrm{OPE} \\
\mathrm{MAC}\end{array}$ & $\begin{array}{l}\text { AATION NAME: } \\
\text { HIacket } \\
\text { HINE USED: }\end{array}$ & Taking & & & & & \\
\hline No & Operation & Obser & $\frac{1 \text { ed Tim }}{2}$ & & $\begin{array}{l}\text { Total } \\
\text { SWVY }\end{array}$ & \begin{tabular}{|l} 
Avg \\
SwV
\end{tabular} & \begin{tabular}{|l|} 
Total \\
SWV
\end{tabular} \\
\hline 1 & $\begin{array}{l}\text { T-shirt front panel and } \\
\text { placket picked }\end{array}$ & 3.2 & 3.5 & 2.8 & 9.5 & 3.17 & 3.17 \\
\hline 2 & $\begin{array}{l}\text { Centre front line cut to } \\
\text { placket length on front } \\
\text { panel }\end{array}$ & 3.1 & 3.1 & 3.5 & 9.7 & 3.23 & 3.23 \\
\hline 3 & $\begin{array}{l}\text { T-shirt front nail pressed } \\
\text { at } 1.5 \text { inch from center } \\
\text { front line panel to inner } \\
\text { side }\end{array}$ & \begin{tabular}{|l|}
6.1 \\
\end{tabular} & 6.2 & 6.1 & 18.4 & 6.13 & 6.13 \\
\hline 4 & $\begin{array}{l}\text { Placket fabric kept in } \\
\text { stitching area and } \\
\text { pressure foot and needle } \\
\text { moved down }\end{array}$ & 2 & 2.5 & 2 & 6.5 & 2.17 & 2.17 \\
\hline 5 & \begin{tabular}{|l}
$\begin{array}{l}\text { Placket stitched on front } \\
\text { panel }\end{array}$ \\
\end{tabular} & 12 & 12.2 & 12.7 & 36.9 & 12.30 & 12.30 \\
\hline 6 & \begin{tabular}{|l|}
$\begin{array}{l}\text { Placket taken out and } \\
\text { turned }\end{array}$ \\
\end{tabular} & 5.8 & 6.5 & 6.7 & 19 & 6.33 & 6.33 \\
\hline 7 & $\begin{array}{l}\text { Top stitch at } 5 \mathrm{~mm} \text { from } \\
\text { edge }\end{array}$ & 15 & 15 & \begin{tabular}{ll|}
15.6 \\
\end{tabular} & 45.6 & 15.20 & 15.20 \\
\hline 8 & $\begin{array}{l}\text { Pressure foot and needle } \\
\text { moved up fabric pulled } \\
\text { out and trimming }\end{array}$ & 2.7 & 2.8 & 2.6 & 8.1 & 2.70 & 2.70 \\
\hline 9 & \begin{tabular}{|l|} 
Turned inside and press \\
nail at 1.5 inch from \\
early folded area from
\end{tabular} & 9 & 9.7 & 10.2 & 28.9 & 9.63 & 9.63 \\
\hline 10 & $\begin{array}{l}\text { Placket fabric kept in } \\
\text { stitching area and } \\
\text { pressure foot and needle } \\
\text { moved down }\end{array}$ & 2.1 & 2 & 2.2 & 6.3 & 2.10 & 2.10 \\
\hline 11 & $\begin{array}{l}\text { Placket stitched back } \\
\text { panel }\end{array}$ & 12.5 & 12 & 12.2 & 36.7 & 12.23 & 12.23 \\
\hline 12 & $\begin{array}{l}\begin{array}{l}\text { Placket turned and } \\
\text { trimmed }\end{array} \\
\end{array}$ & 3 & 3.5 & 3.2 & 9.7 & 3.23 & 3.23 \\
\hline 13 & \begin{tabular}{|l|}
$\begin{array}{l}\text { Top stitch at } 5 \mathrm{~mm} \text { from } \\
\text { edge }\end{array}$ \\
\end{tabular} & \begin{tabular}{l|l|}
15 \\
\end{tabular} & \begin{tabular}{ll|}
14.8 \\
\end{tabular} & \begin{tabular}{l|l|}
14.6 \\
\end{tabular} & 44.4 & 14.80 & 14.80 \\
\hline 14 & \begin{tabular}{|l|} 
Pressure foot and needdle \\
moved up pulling fabric \\
out and trimming
\end{tabular} & 2.7 & 2.8 & 2.6 & 8.1 & 2.70 & 2.70 \\
\hline 15 & $\begin{array}{l}\text { Stitched fabric is kept } \\
\text { left side on the bin }\end{array}$ & 1 & 1.1 & 1.2 & 3.3 & 1.1 & 1.1 \\
\hline & & & & & & Total & 97.03 \\
\hline
\end{tabular}

Calculations:

Standard Minute Value (SMV): $97 \mathrm{sec}$

Calculation of SAM:
Allowance: $15 \%$

SAM (Standard Allowed Minutes) $=$ Basic Time + Total Allowances

$$
\begin{aligned}
& \text { SAM }=(\text { SMV }+15 \% \text { allowance }) \\
& \quad=97+15 \% \\
& =97+14.5 \\
& =111 \mathrm{Sec}
\end{aligned}
$$

Therefore, $\mathrm{SAM}=111 \mathrm{sec}=1.8 \mathrm{~min}$

Maximum output $=($ Working time $/$ Maximum process time $)$ $=(60 / 1.8)$

$=33.33$ Pcs

Productivity per Operator $=$ Total output $/$ Total manpower $=33 \mathrm{Pcs} / 15$ Operator

$=2.2 \mathrm{Pcs} /$ Operator is the output.

\section{h) Time Study Chart for Side Seam}

\begin{tabular}{|c|c|c|c|c|c|c|c|}
\hline & & TIM & STUI & $\mathrm{Y} \mathrm{CH}$ & & & \\
\hline & $\begin{array}{ll}\text { ATION NAME: } & \text { SIDE SE } \\
\text { HINE USED: } & \text { SNLS }\end{array}$ & EAM & & & & & \\
\hline No & Operation & \begin{tabular}{|l|} 
Obse \\
1
\end{tabular} & $\frac{\operatorname{ved~Ti}}{2}$ & $\frac{1 e}{13}$ & $\begin{array}{l}\text { Total } \\
\text { SMV }\end{array}$ & $\begin{array}{l}\text { Axg } \\
\text { SMN }\end{array}$ & \begin{tabular}{|l|} 
Total \\
SMV
\end{tabular} \\
\hline 1 & $\begin{array}{l}\text { Fabric is picked and } \\
\text { assembled }\end{array}$ & 3 & 3.3 & 3.2 & 9.5 & 3.17 & 3.17 \\
\hline 2 & $\begin{array}{l}\text { Kept the panel at the } \\
\text { stitching area and } \\
\text { pressure foot and needle } \\
\text { moved down }\end{array}$ & 4.6 & 5.7 & 6 & 16.3 & 5.43 & 5.43 \\
\hline 3 & $\begin{array}{l}\text { Edge stitch at } 5 \mathrm{~mm} \text { from } \\
\text { edge of the panel }\end{array}$ & 6.2 & 7.3 & 6.8 & 20.3 & 6.77 & 6.60 \\
\hline 4 & $\begin{array}{l}\begin{array}{l}\text { Side label is placed and } \\
\text { pressure foot and needle } \\
\text { moved down }\end{array} \\
\end{array}$ & 2 & 2.5 & 3 & 7.5 & 2.5 & 2.50 \\
\hline 5 & $\begin{array}{l}\text { Edge stitch at } 5 \mathrm{~mm} \text { from } \\
\text { edge of the panel }\end{array}$ & 30 & 32 & 35 & 97 & 32.3 & 32.33 \\
\hline 6 & Side label is stitched & 6 & 7.5 & 8.8 & 22.3 & 7.43 & 7.43 \\
\hline 7 & $\begin{array}{l}\text { Pressure foot and needle } \\
\text { moved up pulling the } \\
\text { fabric out and trimming }\end{array}$ & 6 & 7.2 & 7.5 & 20.7 & 6.9 & 6.67 \\
\hline 8 & $\begin{array}{l}\text { Band is picked from the } \\
\text { side and assembled }\end{array}$ & 3 & 3.3 & 3.2 & 9.5 & 3.17 & 3.17 \\
\hline 9 & $\begin{array}{l}\text { Kept the panel at the } \\
\text { stitching area and } \\
\text { pressure foot and needle } \\
\text { moved down }\end{array}$ & 2 & 2.5 & 2.4 & 6.9 & 2.3 & 2.30 \\
\hline 10 & $\begin{array}{l}\text { Edge stitch at } 5 \mathrm{~mm} \text { from } \\
\text { edge of the panel and } \\
\text { band }\end{array}$ & 6 & 7.5 & 8.8 & 22.3 & 7.43 & 7.43 \\
\hline 11 & $\begin{array}{l}\text { Band tacking is done } \\
\text { and turned }\end{array}$ & 6.5 & 7.2 & 8.3 & 22 & 7.33 & 7.33 \\
\hline 12 & Band stitch finished & 6 & 7.5 & 8.8 & 22.3 & 7.43 & 7.43 \\
\hline 13 & $\begin{array}{l}\text { Pressure foot and needle } \\
\text { moved up and Pulling } \\
\text { the fabric out }\end{array}$ & 2.14 & 2.07 & 3 & 7.21 & 2.4 & 2.40 \\
\hline 14 & Thread trimming & 3 & 3.2 & 3.5 & 9.7 & 3.23 & 3.23 \\
\hline 15 & $\begin{array}{l}\text { Fabric kept on the left } \\
\text { side bin }\end{array}$ & 3.3 & 3.5 & 3.7 & 10.5 & 3.5 & 3.50 \\
\hline & & & & & & Total & 100.92 \\
\hline
\end{tabular}

A time study chart for side seam is shown in Table 15.

Table 15

Time study chart for side seam

\section{Calculations:}

Standard Minute Value (SMV): $100.92 \mathrm{sec}$

Calculation of SAM:

Allowance: $15 \%$

SAM (Standard Allowed Minutes) $=$ Basic Time + Total Allowances

$$
\begin{aligned}
& \text { SAM }=(\mathrm{SMV}+15 \% \text { allowance }) \\
& =100.92+15 \% \\
& =100.92+15.13 \\
& =116.05 \mathrm{Sec}
\end{aligned}
$$

Therefore, $\mathrm{SAM}=116.05 \mathrm{sec}=1.9 \mathrm{~min}$ 
Maximum output $=($ Working time $/$ Maximum process time $)$

$=(60 / 1.9)$

$=31.6$ Pcs

Productivity per Operator $=$ Total output $/$ Total manpower

$=31.6 \mathrm{Pcs} / 15$ Operator

$=2.1 \mathrm{Pcs} /$ Operator is the output.

\section{B. Employee Motivation}

Incentives can be given to employees in order to encourage them to work more effectively. Incentives include attendance bonus, group incentives, and monetary awards, allow flexibility and allowances, giving a chance to lead, work-life balance, helpful services, healthy food etc. A chart of incentive scheme can be prepared with the help of management. It shows various monetary incentives that can be given to employees depending upon their productivity and output. The monetary and nonmonetary incentives can be given to employees in addition to salary on weekly or monthly basis.

\section{Results and Discussion}

The results obtained after implementing the proposed method and employee motivation schemes are discussed.

\section{A. Productivity Improvement}

By following the methodology, the following results are obtained. The results are measured and shown in the form of graphs for better understanding.

\section{1) Operation Breakdown Chart for Placket and Side Seam}

The following graph shows the comparison of the operation breakdown steps between the original method and proposed method for placket and side seam finishing.

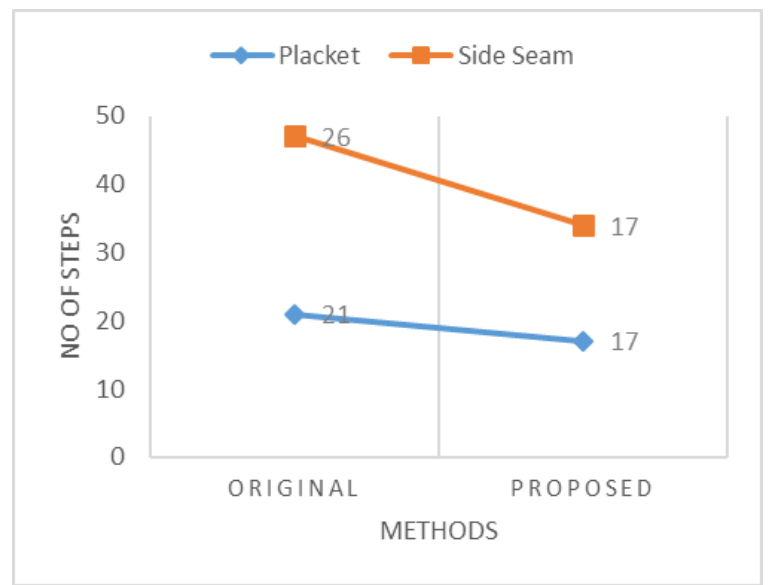

Fig. 6. Operation breakdown for placket and side seam

From Figure 6, it is observed that the operation breakdown steps are decreased from 21 to 17 for placket and from 26 to 17 for side seam. Time and motion study is carried out by combining various operations and thus the number of operations are reduced.

2) Two Handed Process Chart for Placket and Side Seam

The following graph shows the comparison of the two handed process chart between the original method and proposed method for placket and side seam finishing.

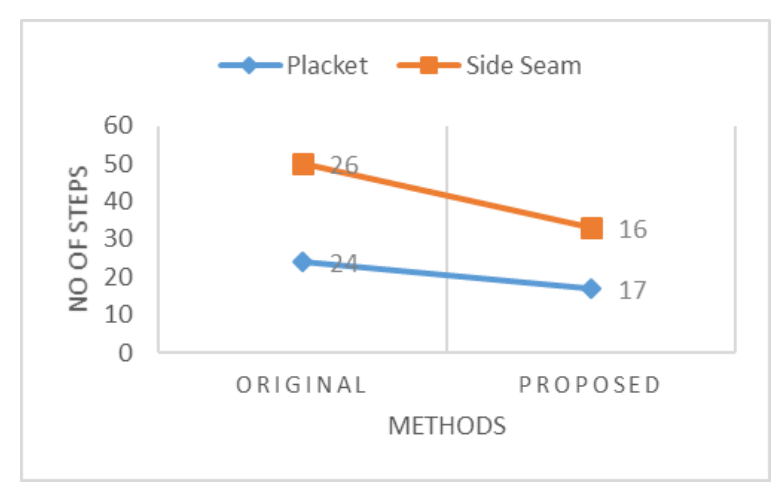

Fig. 7. Two handed process chart for placket and side seam

In this graph its clearly shows that the hand movements are decreased from 26 to 16 for placket and 24 to 17 for side seam. When we do the time and motion study we are combining various operations for both hands and thus the number of motions for both right and left hand motions are reduced.

\section{3) Time Study Chart for Placket and Side Seam}

The following graph shows the comparison of the time study chart between the original method and proposed method for placket and side seam finishing.

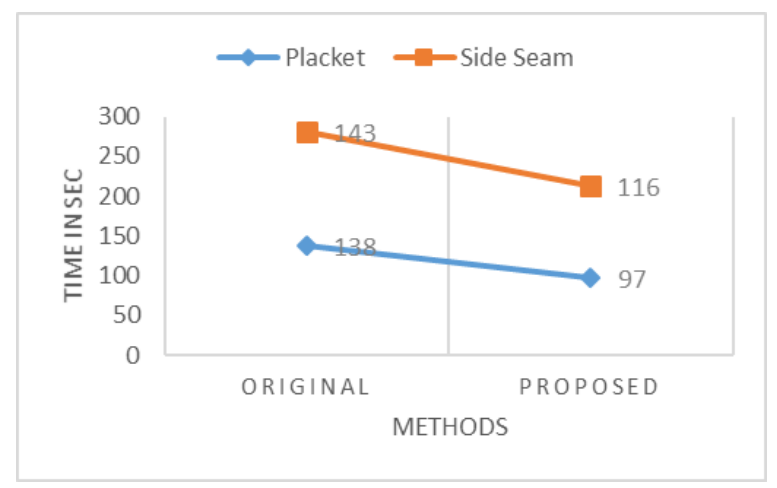

Fig. 8. Time study chart for placket and side seam

From Figure 8, it is observed that the time taken to complete the operation is comparatively reduced in the proposed method. This is because of following the time and motion study by combining various operations.

4) Output per Hour before and After Motion Study - Placket

Figure 9 shows the comparison of the output per hour before and after motion study for placket and side seam.

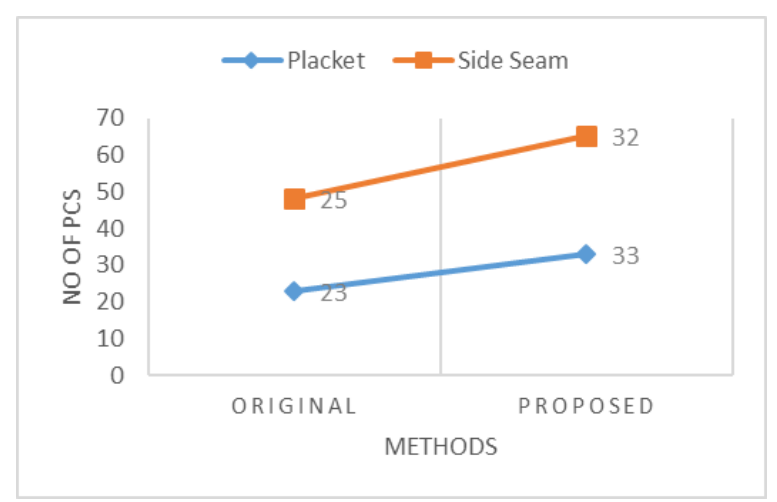

Fig. 9. Output per operator for placket and side seam 
From the Figure, it is observed that the output per hour for each operator for both placket and side seam in original method is lower than the proposed method. As we can see that the output is increased dramatically after we implemented the time and motion study.

5) Percentage of Efficiency Before and After Motion Study

The following Figure 10 shows the percentage of efficiency before and after motion study for placket and side seam.

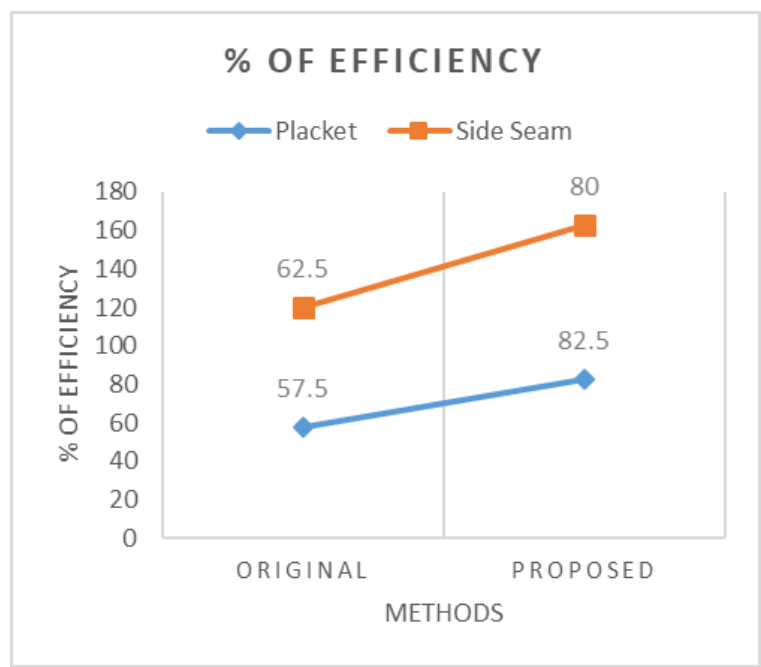

Fig. 10. Percentage of efficiency before and after motion study

It is observed that the efficiency percentage of both the operations is increased dramatically after the implementation of the new proposed method.

\section{B. Employee Motivation - Incentive Schemes}

A chart of incentive scheme is prepared with the help of management. It shows various monetary incentives that can be given to employees depending upon their output. The monetary and non-monetary incentives are given to the employees in addition to salary on weekly or monthly basis.

\section{1) Attendance Bonus}

Various monetary incentives can be awarded to the employees who maintain good and regular attendance. Figure 11 shows the attendance bonus extended to the employees at different period.

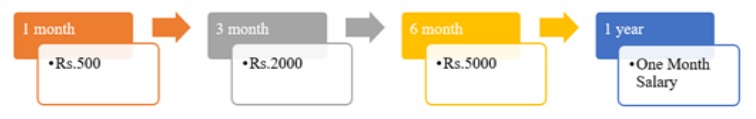

Fig. 11. Attendance bonus

With the help of management, the employees who holds full attendance for one month is awarded with Rs.500, for three months Rs. 2000. For a period of 6 months, the bonus is Rs. 5000. If they maintain full attendance for one whole year they will be awarded with one-month salary in order to motivate them to work more efficiently.

\section{2) Group Incentives}

In order to promote the group activities and team work the monetary incentives can be awarded based on the whole team achievement. Figure 12 shows the group incentives extended to the employees.

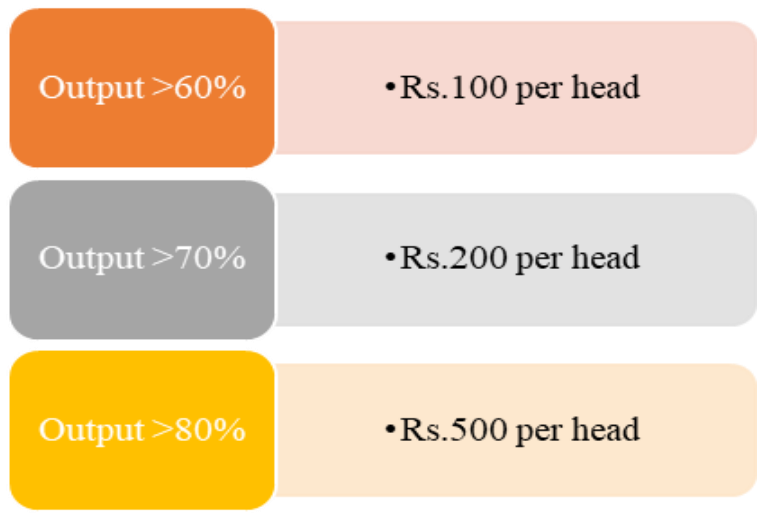

Fig. 12. Group incentives

The employees are divided into various teams depending on their type of operations carried out in the industry. Based on the productivity achieved by each team the incentives are given by the management to promote team work and to increase healthy competition between the teams. The team who achieves output efficiency above $60 \%$ is awarded with Rs. 100 per head in the team. For output efficiency above $70 \%$, the team is awarded with Rs. 200 per head and for output efficiency above $80 \%$, each member in the team is awarded with Rs. 500. Through the incentive schemes, the team work is enhanced and employees are motivated.

\section{3) Allowances}

Employees can take leave any day they want. But the days should not exceed the allowance of $10 \%$ which is 3 days a month; with prior notice, they will be awarded leave with salary. Exceeding 3 days without prior notice will result in cutting salary for one day extra.

Experienced employees are assets of any industry. In order to control switching of industries and to keep permanent labor within the premises, extra allowances for long job tenure were suggested and management accepted to extend this allowances to well experienced employees. Figure 13 shows the monetary awards extended to employees. For operators with 3 years' job tenure, an award of Rs. 36000 was extended at the end of third year along with Diwali bonus for better use. Employees with more than five years of experience, an amount of Rs. 60000 is awarded as a bonus. After this the entire cycle is repeated again.

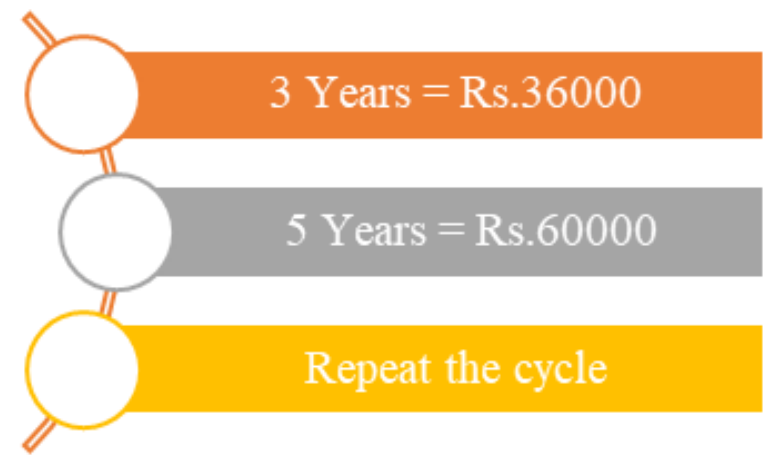

Fig. 13. Monetary awards 
To encourage the working women, a scheme "Gold for Marriage" was suggested. This award was extended to women who are efficient and good performers in the company. Figure 14 shows the gold for marriage scheme. If the employee works in the company for 1 year, 2-gram gold will be awarded. 4 grams for 2 years and for women working in the company for about 4 years, 6-gram gold will be awarded. This method increases the trust and good relationship between the employees and the management.

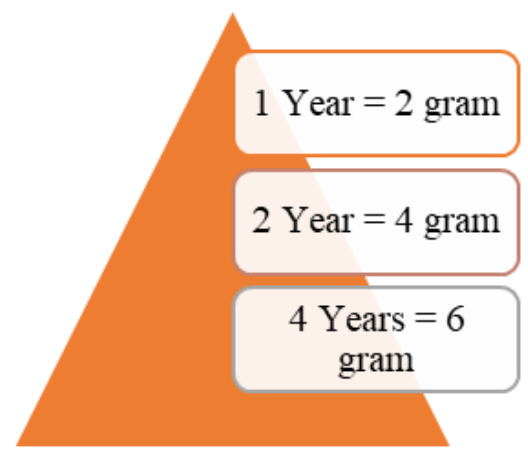

Fig. 14. Gold for marriage

\section{4) Chance to Lead}

Weekly once any one person is chosen from the group to lead the group activities. They will assist the supervisor in all matters. During the supervisor's absence in the company, the group leaders will take up the responsibility and guide the rest of the people in the group. In this way, all the members in the team are motivated and gets a chance to lead and every member in a team will be aware of entire work and their responsibilities.

\section{5) Helpful Services}

Opening the bank account and depositing the salary in bank for all staffs were extended to the employees who work in the industry for more than 6-month time period. Opening savings account and deposit $5 \%$ of their salary separately which helps them to meet the immediate and unforeseen expenses.

\section{6) Work-Life Balance}

A small day trip is arranged once in every 4 months including all staffs and labors who work within the company. The employees were permitted to bring their family. This will improve the work life balance.

\section{Conclusion}

Production is one of the most important department in any industry, as it plays a major role in deciding the profit or loss. Therefore, the study was carried in an apparel industry in Tiruppur to increase the productivity without disturbing the input investment. It is challenging to identify the reasons for finding out why productivity is not meeting the planned target even though everything is planned and executed according to the buyer's requirement. The study carried out by analyzing the productivity and the methods to improve the productivity through time and motion study. It is found that the time study, altering the operations, reducing the waiting time of the machine, helps in improving the efficiency of operators in the line, which in turn resulting in increased production.

Employee motivation plays greater part in increased production. As a result, various incentive schemes are introduced to motivate the employees based on their efficiency, sincerity and productivity.

Thus, the productivity in sewing department is improved by using work study method. The various process and procedures are improved and numerous steps are reduced by using motion study. The efficient use of man machine and materials is achieved by time and motion study. The employees are well motivated.

\section{References}

[1] A. Rajiwate, H. Mirza, S. Kazi, and M. M. Momin, "Productivity Improvement by Time Study and Motion Study", in International Research Journal of Engineering and Technology, vol. 7, no. 3, March 2020.

[2] A. K. Singh, A. Kumar, and A. Chakravarty, "Method Study and Time Study", pp. 6-30, Nov, 2020

[3] G. Kanawaty, "Work Study (Time and Motion Study)", Introduction to Work Study, Geneva International Labor Office, 1955, ch. 7, sec. 1 pp. 171-203.

[4] Glock and Kunz, Apparel Manufacturing: Sewn Product Analysis, 4th Edition, Pearson, 2005, p. 68.

[5] V. Ramesh Babu, "Productivity", Industrial Engineering in Apparel Production, 1st Edition, Wood head Publishing India, 2011, p. 5.

[6] https://textilestudycenter.com/

[7] https://www.onlineclothingstudy.com/ 